\title{
Simulated Annealing and Direct Search based Optimization Models for Facility Location in Logistic Network Design
}

\author{
Shaju Varughese \\ Research Scholar, Bharathiar University, \\ Coimbatore, Tamilnadu, India
}

\author{
Gladston Raj S. \\ Head, Department of Computer Science, \\ Govt. College, Nedumangadu, \\ Thiruvananthapuram, \\ Kerala, India.
}

\begin{abstract}
Finding the optimum location of facilities is an important problem related in logistics. Locations of Distribution Centers (DCs) can be at the proximity of highways and closer to populated areas in order to speedup package deliveries and minimize the overall transport cost and time. Deciding the optimum number of DCs as well as the optimum location of DCs are the most important aspects of the design of any logistics network. Deciding the number of DCs will depend upon the budget and optimum locations of DCs will reduce the overall transport cost. As operations globalize, location decisions become more complex. A powerful approach to analyzing these problems is the transportation method of linear programming. The linear programming based methods will take much time to attain a solution in such a wide problem space. But for dealing with such wide problem space, soft computing based approaches are well suited and can find a meaningful solution in finite time. This work generate the Simulated Annealing and Direct Search based model for facility location in logistics and evaluate its performance. Also, compare the performance of this model with a k-mean clustering based model.
\end{abstract}

\section{Keywords}

Optimization, clustering, logistics, Euclidean distance, annealing, K-means, Direct search, maximizing, minimizing, facility location

\section{INTRODUCTION}

The facility location analysis is the process identifying the location problem and generates a common model for finding the optimal location. This is nothing but identify $\mathrm{k}$ center problem, which deal with the optimal placement of facilities to minimize transportation costs by satisfying some constraints. The techniques also apply to cluster analysis so that the clustering algorithms used in data mining can also be applied for location analysis. In this work, we design a soft computing based model for facility location for logistics analysis.

\subsection{Facility Location Problem (FLP)}

Facility Location identification and allocation problem point to the solutions of finding the optimal/best solution to construct one or more facilities in order to attend the largest subset of users within a service distance. The facility location problem is a challenging and non-linear problem in the areas of production, procurement, distribution, operations management and combinatorial optimization. The problem finds an optimal location of facilities considering facility construction costs, transportation costs, etc. This problem is very popular because it is faced by majority of companies.
Location models are very difficult to solve, especially for large heterogeneous problem instances[3]. There are a number of specific tools that are available in the operational industry that overcome the tedious computational complexity of a location models. So, it is very difficult to develop a common model that can customize to a particular location model that is optimal for all potential or existing applications [3].

There are two types of techniques in general[3]
a) Exact Solution Techniques[3]
b) Heuristic Solution Techniques[3]

\subsection{Applications of FLP}

The research in this area has not only concentrated on the mathematical modeling and algorithmic aspects but also in the area of innovative applications. The applications include various optimization areas such as parameter calibration, land use optimization, transport route searching, retail shop outlet location selection, spatial objects optimization, performance optimization of urban modeling. When applied to telecommunication network design and capacity planning, the difficult task of designing and maintaining huge, reasonably optimal, telecommunication networks becomes more feasible. Many major telecommunication service providers are finding it imperative to upgrade or expand their facilities and services. The facility location models can make use of the design of various types of telecommunication networks. The capacitated optimization technique can play an important role for the improvement of the telecommunication network design. In this example, most of the analysis decisions have to be made concerning relation between concentrator location and cable expansion. Many analysts have claimed that more than 50\% of capital and operating costs are a consequence of the provision of Local Access Telecommunication Networks $(\mathrm{LATN})[$ ].

\subsection{The FLP and Logistics}

\subsubsection{Logistic}

An efficient form of information planning for business to create the framework for the flow of products is called logistic. The suppliers and customers co-ordinate and generate the logistic structure in supply chain management and seeks to achieve connectivity and co-ordinate between the processes of other events in the system. The cost of performing logistics is a major expenditure for most businesses and supply chain arrangements. In logistic management the actual locations must be established to form a transaction network and to the extent required, warehousing, materials handling, and packaging activities must be performed. Because the work of logistics is extremely detailed and complex, there is a natural tendency to focus on functional performance. The functions of 
logistics combine the three. The primary operational processes of logistics are customer accommodation, procurement, and manufacturing support.

Optimization is the process of minimizing or maximizing the output of a well defined functions model. This problem tries to satisfy the fixed, presumably known demands of $\mathrm{m}$ customers by supplying each customer with the same commodity from a facility to be established at a particular site $\mathrm{j}$. It is assumed that there are $\mathrm{n}$ candidate sites where facilities can be established if necessary. The unfortunate aspect of this very general class of mathematical programming problems is the fact that many of these problems have a high degree of complexity and general purpose algorithms to solve them dependably are not readily available. The continuing deployment of more powerful computer and software platforms is making the feasibility of finding exact solutions to large problems in this area possible. Future, the modern soft computing based approaches are capable of searching an optimum solution in the wide problem space and will be much helpful to attain a meaningful solution.

\subsubsection{Facility Network Design}

Classical economics neglected the importance of facility location in overall network design for efficient business operation is not well defined in classical economics. The economists originally discussed supplier and customer demand relationships, facility location and transportation cost were either nonexistent or equal among competitors. In business management, the number, size, and geographical relationship of service providers used to perform logistical operations directly affect the customer service.

\subsubsection{Logistics Network}

A logistics network provides cost effective and efficient way of facilitates delivery of packages. It is a network of DCs which can be utilized by multiple organizations. These organizations can share associated logistics costs. Consolidation of a variety of products and truckload transportation help for mitigation of traffic congestion, saving of fuel, and reduction in labor costs. Use of advanced information systems in the PLN can improve coordination in different stages of supply chain minimizing uncertainties. A network of public distribution centers would be constructed as mid-stage between retailers and customers.

Locations of DCs can be in proximity of highways and closer to populated areas in order to expedite package deliveries. The type of products that can be handled by these DCs include furniture, consumer electronics, paper products and stationary items, drugs, textile products, perishable and non-perishable food products, non-food grocery products, home improvement and hardware items. Since these products include almost everything related to domestic and office needs, implementation of the PLN can reduce daily vehicle trips for purpose of shopping by substantial amount. Moreover, not only retailers, wholesalers and manufacturers can use of PLN but third party logistic companies working with cooperative contract, small and mid-sized freight transportation enterprises can collaborate with PLN and reduce the costs with high quality service to their customers.

The working principle of a public logistics network is very similar to the Internet. The Internet is a network of routers. When a user requests for a data packet, the request is transferred to the respective server. The data packet sent by a server travels through series of routers in the network before reaching to the user. Furthermore, the Internet is not owned by any organization, while organizations connect their own local network to the Internet.

Routers and data packets in the internet are analogous to DCs and packages in a PLN, respectively. Additionally, the entire PLN is not owned by any single organization. There are some organizations having their own established logistics network in the market which can make their business operations very cost-effective. However, not all organizations may be able to afford to build their own network because the capital cost for constructing logistics network infrastructure is too high. Utilizing the features of a PLN could help all the companies in the network reduce their costs and improve freight-market competitiveness.

\section{Background on Facility Location and About this Work 2.1 Facility Location Problem}

The facility location problem an optimal location of facilities considering facility construction costs, transportation costs, etc. This is a common problem and required adequate solution for the smooth functioning of the industry and thus the companies. There are number of contributions for the solution of this problem and also number researches have been conducted.

The purpose of this work is to build a decision support system that helps the managers to decide where to locate a facility.

Now consider the general definition of facility location problem.

Let $\mathrm{I}$ and $\mathrm{J}$ denote a set of facilities and customers, respectively $(i=1, \ldots, m$ and $j=1, \ldots, n)$, and let fi indicates the actual cost of locating a facility at particular location i. Let cij be the cost of supplying customer $\mathrm{j}$ from facility $\mathrm{i}$. The decision variables are as follows:

xij: denotes the fraction of customer $\mathrm{j}$ 's demand satisfied by facility $i$

$$
y_{i}= \begin{cases}1 & \text { if it locate a facility at location } i \\ 0 & \text { otherwise. }\end{cases}
$$

Time complexity of problems of the above mentioned problem is an obvious one, so that linear programming based methods will take much time with respect to the size of the problem and the constraint. In this work, it will design a soft computing model for solving a simple facility location problem with minimum constraints.

\subsection{The Proposed Logistics Network Design}

In Bansal [2], a Public Logistics Network (PLN) for the continental U.S. was designed. This research uses the same design approach that was developed by Bansal. Bansal's design can be explained as four step process that includes generation of the Underlying Road Network (URN), developing the network of public DCs, estimation of average package delivery time, and finding public DC locations that minimize average package delivery time.

In this work, the PLN will be designed using a similar process with some modifications and simplification in the steps. In this work, it use a simplified version of that design so that, instead of using the "average package delivery time" as a metric for optimization, model used simple distance as the metric in the fitness function of the soft computing model. This approach was used to minimize the optimization time. 
Since the distance is directly proportional to package delivery time, believe that this approach also will lead to equal results, logically with in lesser time.

- Generation of the Underlying Road Network (URN)

- Developing the network of DCs,

- Finding public DC locations that minimize the distance between the DCs and the User locations.

\subsubsection{Generation of the Road Network of USA}

The following map shows the road network that created from the US census data set.

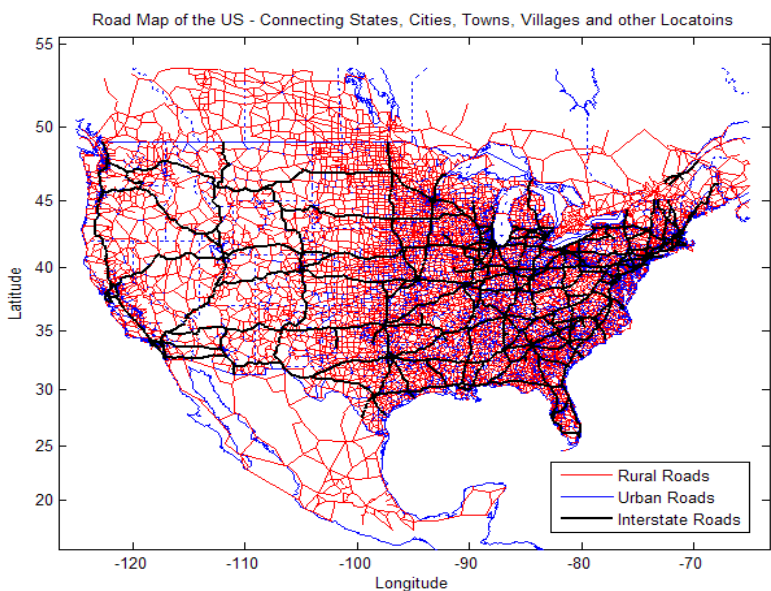

Figure 1. Road Network of USA

\subsubsection{Generation of the Underlying Road Network of Regional Distribution Centers.}

The population in RDC is represented by total 925 U.S. census blocks that are plotted on the map of RDC. A subgraph of the road network was generated that is then followed by the removal of two-degree nodes from the network. Each point in this network is a potential location for a DC.

The following graph/map shows the road network of Louisiana, USA that will be the example of a sub graph created and used to create the regional distribution centers (RDC) that are interested in.

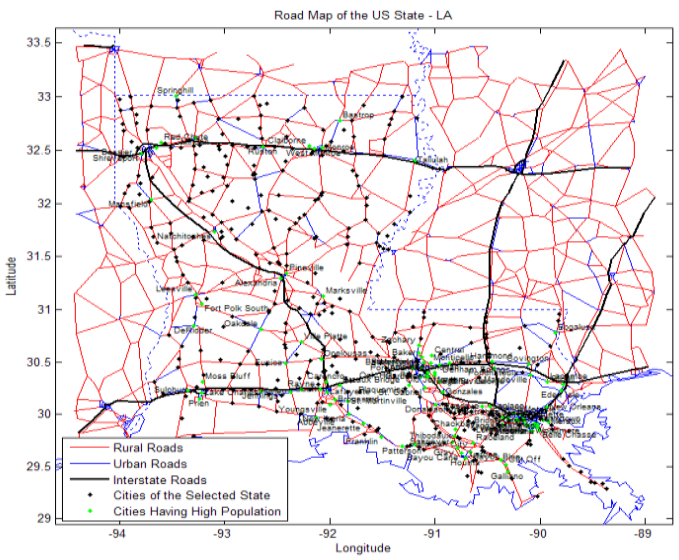

Figure 2. Underlying Road Network of RDCs

\subsubsection{Network of DCs}

If needed, it may also create the network of DCs as follows. The arcs connecting census blocks to the URN are added to the network and the shortest time paths and distances between each pair of points are calculated using Dijkstra's algorithm. DCs will be located at some of the key points and then connected to each other using Delaunay Triangulation [19] to form a network of public DCs. The shortest time paths between all pairs of DCs is found and those paths and distances are then used to calculate the percent flow of the packages, wij from DC $i$ to DC $\mathrm{j}$ using order based proximity factors developed by Kay and Parlikad [1] using following equations.

$$
\begin{aligned}
& w_{i[j]}^{\prime}=w_{i[j]}^{0} \cdot \frac{\frac{p}{m}\left(1-\frac{p}{m}\right)^{(j-1)}}{\frac{1}{m} \sum_{k=1}^{m} \frac{p}{m}\left(1-\frac{p}{m}\right)^{(k-1)}}=w_{[\lfloor j]}^{0} \cdot \frac{\left(1-\frac{p}{m}\right)^{(j-1)}}{\frac{1}{m} \sum_{k=1}^{m}\left(1-\frac{p}{m}\right)^{(k-1)}} \\
& w_{i[j]}=\frac{w_{i[j]}^{\prime}}{\sum_{k=1}^{m} \sum_{l=1}^{m} w_{k l}^{\prime}}
\end{aligned}
$$

In this work, apply simulated annealing and Direct Search to find optimum location of DCs based on the local distance between the DCs and the User locations.

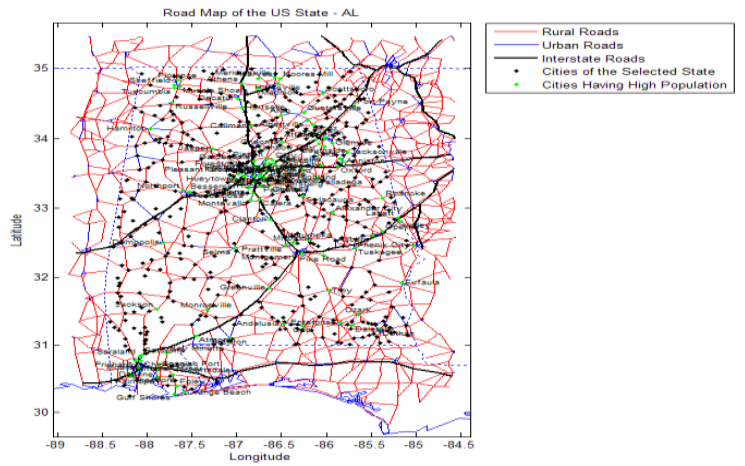

Figure 3. Map : Alabama, USA

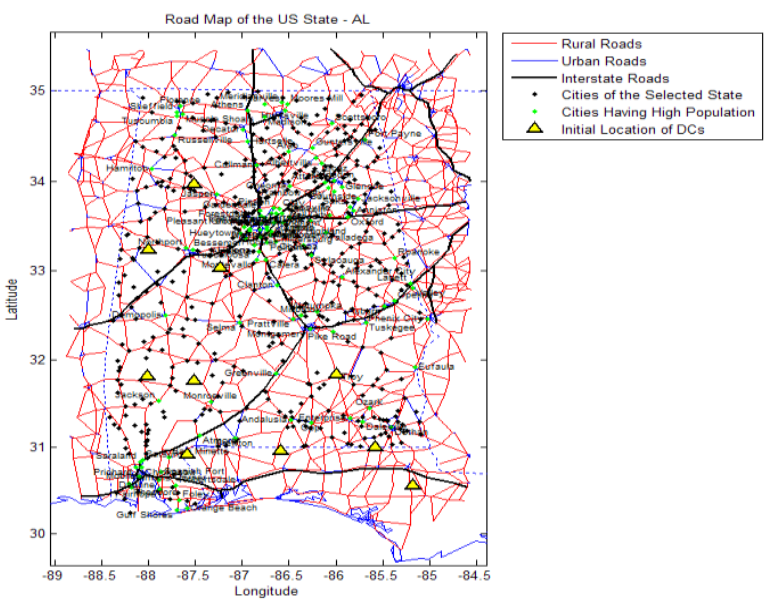

Figure 4. Random Initial Locations of DCs

\section{MODELING THE SOFT COMPUTING BASED FACILITY LOCATION SYSTEM}

\subsection{Simulated Annealing}

The cooling of materials is the concept and the working terminology of Simulated Annealing(SA). The Metropolis algorithm developed in 1953 was the basic structure of Simulated Annealing and it was published. The algorithm in this paper simulated the cooling process of materials and their 
structural changes from their heavy hot stage by using the heat bath. This cool down process is known as annealing. When we heat a material(solid) up to the melting point and then allow to cool it, the structural qualities of the solid depend on the cooling rate. Large number of crystals will be formed, if try to cool the liquid. But, if try to cool the liquid in a single step, we will not get the perfect crystals. The Metropolis's algorithm will generate a model and represent the material as a system of particles. The working of the algorithm is that in step by step it will allow the material to cool and transform in to a steady solid state.

The idea of metropolis algorithm has initiated by Kirkpatrick in 1982. It has been applied to various optimization problems. The basic concept is to find the feasible solution to a problem by using simulated annealing as a major tool and converge to an optimal solution. Simulated annealing is described in many textbooks. Dowsland in 1995, layout the core concept of simulated annealing in simple manner.

The law of thermodynamics state that at temperature, $t$, the probability of an increase in energy of magnitude, $\delta \mathrm{E}$, is given by $\mathrm{P}(\delta \mathrm{E})=\exp (-\delta \mathrm{E} / \mathrm{kt})$

Where $\mathrm{k}$ is a Boltzmann's constant.

The simulation in the Metropolis algorithm calculates the new energy of the system. Based on the calculated new energy The system has moved to the same state if the new energy has decreased otherwise the new state is accepted using the probability returned by the above formula. A certain number of iterations are carried out at each temperature and then the temperature is decreased. This is repeated until the system freezes into a steady state. This equation can make use for generating simulated annealing concept directly. It is a common practice to drop the Boltzmann constant into the equation to experiment with different materials. The acceptable probability for the worse case is defined by the equation

$$
\mathrm{PR}=\exp (-c r / t r)>\mathrm{rd}
$$

Where

$$
\begin{aligned}
& c r=\text { the change in the evaluation function } \\
& t r=\text { the current temperature } \\
& r \mathrm{~d}=\quad \text { a value between } 0 \text { and } 1
\end{aligned}
$$

The probability of accepting a worse move is a function of both the temperature of the system and of the change in the cost function. This was shown in the lectures and a spreadsheet is available from the web site for this course which shows the same example that was presented in the lectures. That is as the temperature of the system decreases the probability of considering a worse move is also decreased. This is similar to the gradual movement to a frozen state in actual physical annealing. Similarly the temperature is zero then only better moves will be accepted.

\subsection{Direct Search}

The optimal solution generating procedures in multidimensional spaces are some extend similar to the methods used in finding optimal solution in a single dimension. The trade-off between general applicability versus computational complexity also exists in multidimensional optimization.

The most optimal solution to an optimization problem in multidimensional space is depending upon the final objective choose (minimum or the maximum). Even if the solution space is relatively small, this solution approach is a very generally applicable and useful. However, the number of independent variables as well a the dimensions of the problem space, increase, the computational complexity of this solution approach very quickly becomes unmanageable. Therefore, it can find optimum solution without considering all possible solutions by using the relevant methods that intelligently search through the solution space.

It is important to note that some of the popular optimization techniques you may have heard of such as simulated annealing, tabu search, neural networks and genetic algorithms all belongs to the category of optimization techniques.

Function S-ANNEAL(Prob, Sched) will returns a solution state

$\begin{array}{ll}\text { Inputs } & : \quad \text { Prob, a problem } \\ & \text { Sched, a mapping from } \\ \text { time to temperature } & \end{array}$

Local Variables : $\quad$ Currt, a node

Next, a node

S-A-T, a "temperature" monitoring the probability value of downward Stages

Currt $=$ MAKE-NODE $($ INIT-STATE $[$ Prob $])$

For $\mathrm{t} 1=1$ to $\infty$ do

$\mathrm{S}-\mathrm{A}-\mathrm{T}=$ Sched $[\mathrm{t} 1]$

If $\mathrm{S}-\mathrm{A}-\mathrm{T}=0$ then return $\mathrm{Currt}$

Next $=$ a randomly selected successor of Currt

$\Lambda \mathrm{E}=\mathrm{VAL}[$ Next $]-\mathrm{VAL}[$ Currt $]$

if $\Lambda \mathrm{E}>0$ then Currt $=$ Next

else $C u r r t=$ Next only wit(2probability $\exp (-\Lambda \mathrm{E} / \mathrm{S}-\mathrm{A}-\mathrm{T})$

The parameters of the algorithm schedule(sched) and problem(prob). The cooling schedule is structured (Ref. below). This algorithm has been executed the annealing process until the S-A-T reaches to zero. At the time of the implementation by reducing gradually until some other condition is met. The procedure by which this algorithm is executed may make an interrupt in another aspect of the algorithm that is shown more directly in some other applications. That is, a particular phase of the search normally continues at a level of the temperature until some level of equilibrium is attained. This might be a certain number of iterative procedure or it could be until it has been no change in the state for a certain number of iterations. This is all part of the cooling schedule which, in the above algorithm, may hide some of these details.

The four major stages (cooling stages) of a simulated annealing algorithm are

- Initial/Starting Temperature

- Optimal solution based final Temperature

- Decrement pattern of the temperature

- Scheduled iterative procedure at each level of temperature 


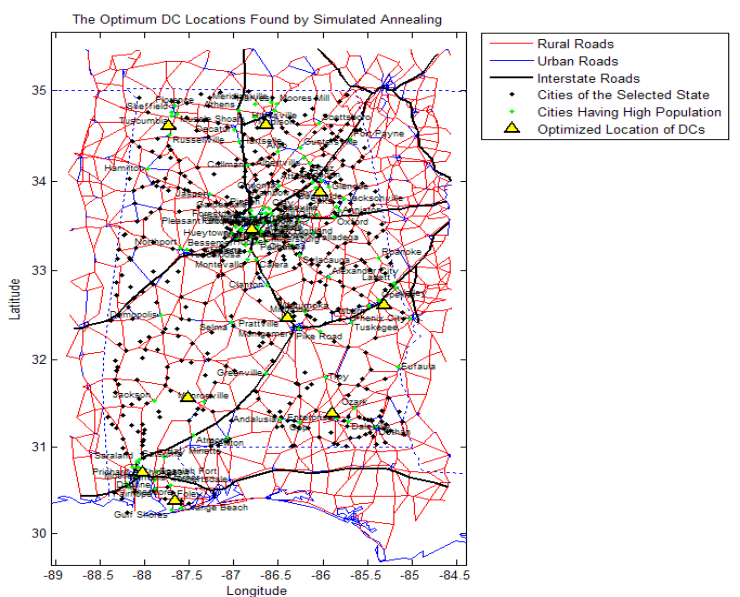

Figure 5: Facility Location by Simulated Annealing

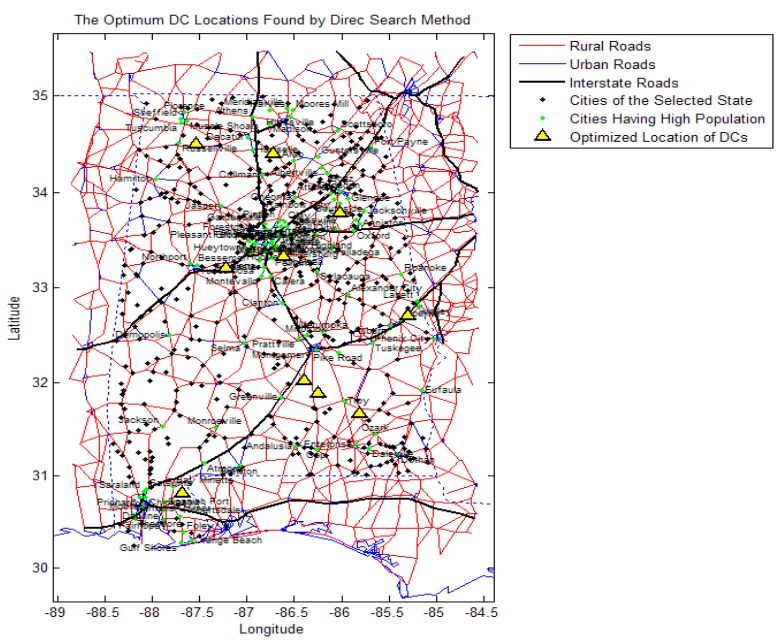

Figure 6: Facility Location by Direct Search
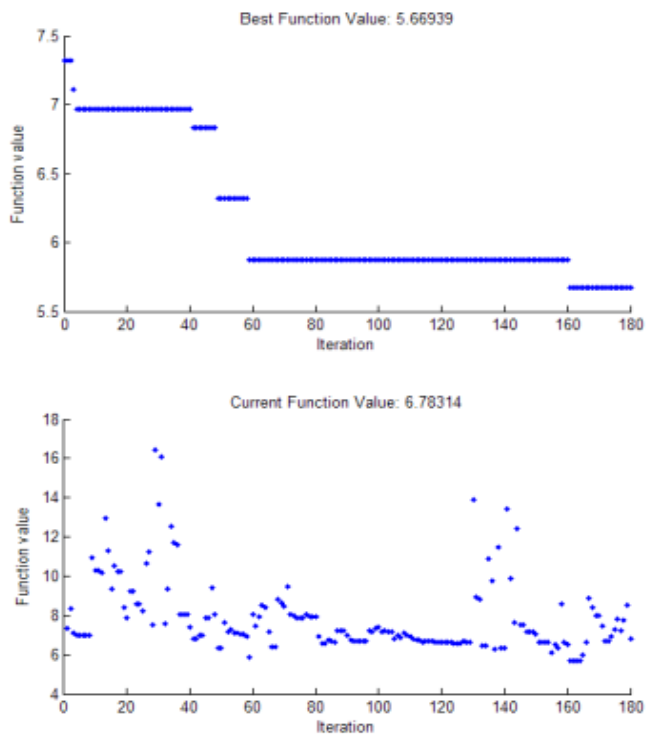

Figure 7: Performance of Simulated Annealing

\subsubsection{Fitness Function}

The following function is used to find the fitness at the set of facility location $\mathrm{XYF}=(\mathrm{Xi}, \mathrm{Yj})$, where $\mathrm{i}, \mathrm{j}=1$ to $\mathrm{n}$. The set of points which has the lowest fitness value will be the optimum location for placing facilities. XYU is the locations of all the customers(cities, towns, villages)

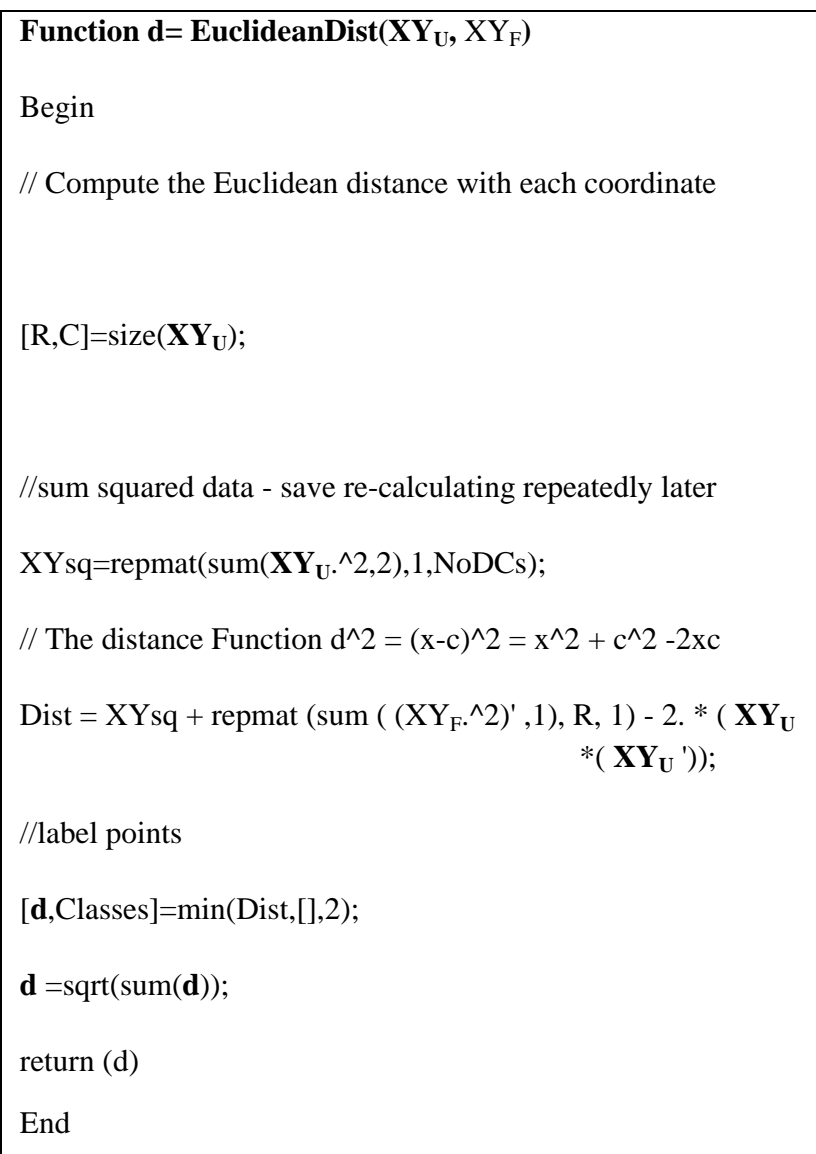

\subsection{K-Means Clustering}

Simply put, k-Means Clustering is an algorithm among several that attempt to find groups in the data. In pseudo code, it follow this procedure:

Initialize $\mathrm{mi}, \mathrm{i}=1, \ldots, \mathrm{k}$, for example, to $\mathrm{k}$ random $\mathrm{xt}$

Repeat

$$
\begin{aligned}
& \text { For all } \mathbf{x}^{\mathrm{t}} \text { in } \mathrm{X} \\
& b_{i}{ }^{t} \leftarrow 1 \text { if }\left\|x^{t}-\mathbf{m}_{i}\right\|=\min _{j}\left\|\mathbf{x}^{\mathrm{t}}-\mathbf{m}_{\mathrm{j}}\right\| \\
& \mathrm{b}_{\mathrm{i}}{ }^{\mathrm{t}} \leftarrow 0 \text { otherwise }
\end{aligned}
$$

For all $\mathbf{m}_{\mathrm{i}}, \mathrm{i}=1, \ldots, \mathrm{k}$

$\mathbf{m}_{\mathrm{i}} \leftarrow$ sum over $\mathrm{t}\left(\mathrm{b}_{\mathrm{i}}{ }^{\mathrm{t}} \mathbf{x}^{\mathrm{t}}\right) /$ sum over $\mathrm{t}\left(\mathrm{b}_{\mathrm{i}}^{\mathrm{t}}\right)$

Until mi c

The vector $m$ contains a reference to the sample mean of each cluster. $\mathrm{x}$ refers to each of our examples, and $\mathrm{b}$ contains estimated class labels.

Explained perhaps more simply in words, the algorithm roughly follows this approach:

1) Choose some manner in which to initialize the $m_{i}$ to be the mean of each group (or cluster), and do it.

2) For each example in your set, assign it to the closest group (represented by $\mathrm{m}_{\mathrm{i}}$ ).

3) For each $m_{i}$, recalculate it based on the examples that are currently assigned to it. 
4) Repeat steps $2-3$ until $m_{i}$ converge.

Now that it has some rudimentary understanding of what kmeans is, what are some practical applications of it?

\section{RESULTS AND DISCUSSION}

It has implemented the proposed soft computing based models for facility location in logistics analysis using Matlab software version R2012s. Also, has used some of the functions of Logistics Engineering Toolbox in this research. It tried to use almost equal input parameters for each and every evaluated method. It used the USA census data and map data which is much suitable for this kind of research. It has decided to use USA data because, it is the only data refereed in some of the previous works and there seems no such detailed data available for any other country for validating the methods of facility location and logistics analysis.

\subsection{The Parameters of the Soft Computing Models}

$\begin{array}{ll}\text { 4.1.1 Simulated Annealing Options } \\ \text { MaxIter } & : 200 \\ \text { MaxFunEvals } & : 200 \\ \text { InitialTemperature } & : 100 \\ \text { AnnealingFcn } & : \text { Fast Annealing } \\ \text { HybridFcn } & : \text { fminsearch } \\ \text { TemperatureFcn } & : \text { temperatureexp }\end{array}$

\subsubsection{Pattern Search Options}

$\begin{array}{ll}\text { MaxIter } & : 200 \\ \text { MaxFunEvals } & : 200 \\ \text { InitialMeshSize } & : 1.0 \\ \text { PollMethod } & : \text { MADSPositiveBasis2N } \\ \text { SearchMethod } & : \text { ADSPositiveBasis2N }\end{array}$

\subsubsection{K-means Clustering}

$\begin{array}{ll}\text { MaxRepetitions } & : 200 \\ \text { MaxIter } & : 20\end{array}$

The following table shows the overall results of this work. Since the performance of a soft computing model will depend up on several factors, and some random conditions, it run each algorithms several times and only selected the values which are minimum.

Table 1 - The Overall Performance

\begin{tabular}{ccc}
\hline $\begin{array}{c}\text { Facility Location } \\
\text { Method }\end{array}$ & Avg. Distance & Time Consumed \\
\hline Random Location & 9.44 & - \\
SA & 5.67 & 5.13 \\
DS & 4.29 & 1.44 \\
k-means & 3.95 & 3 \\
\hline
\end{tabular}

The following graph shows the performance of the four algorithms in terms of the minimum average distance achieved. The average distance is the average of distance between all DCs and the Customer locatoins. Each Customer is bound with a nearest DC and the distance between each customer to that corresponding DC is calculated and then the average of all such distances were calculated. In this graph, the first column shows the reference distance which is nothing but the initial average distance of DCs that are randomly placed on the map.

Among the two soft computing based models, with respect to the average distance, the DS based model performed well. It means, DS found the optimum facility locations better than the other compared methods. But the k-means based clustering performed good. But, during out repeated tests, it found that SA competed the other two in some cases. The performance was very much depend on the parameters of SA.

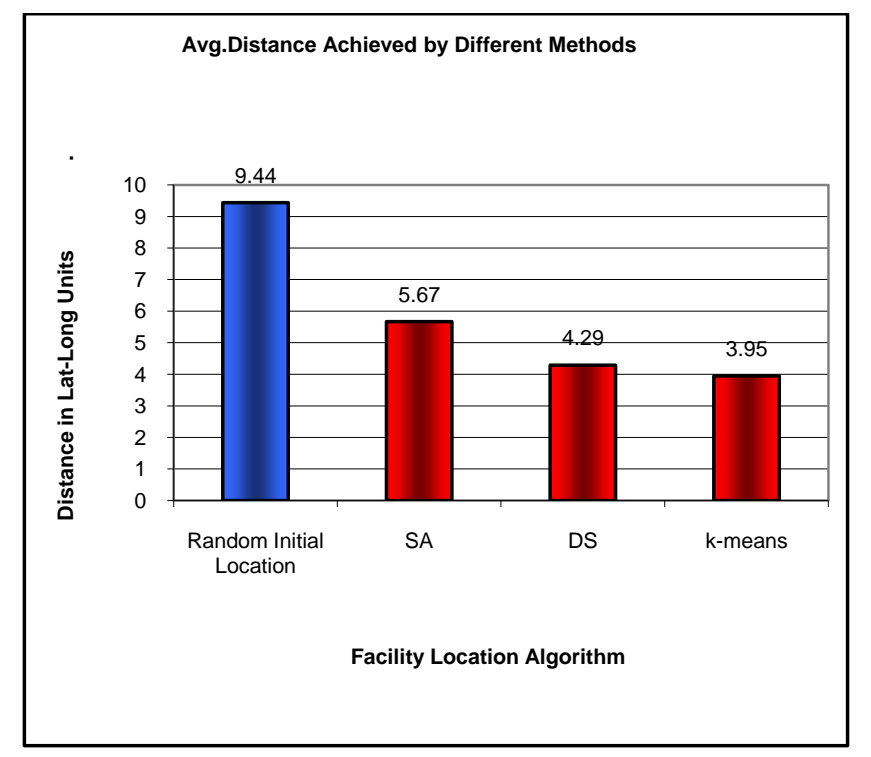

Figure 8. The Performance in terms of distance

The following graph shows the performance in terms of cpu time. The direct search consumed lesser time. It has to note that the time in the case of DS and SA includes some additional time that is spent of displaying/updating some of the performance graphs also (without those performance graphs, there will be much reduction in time in SA and DS)

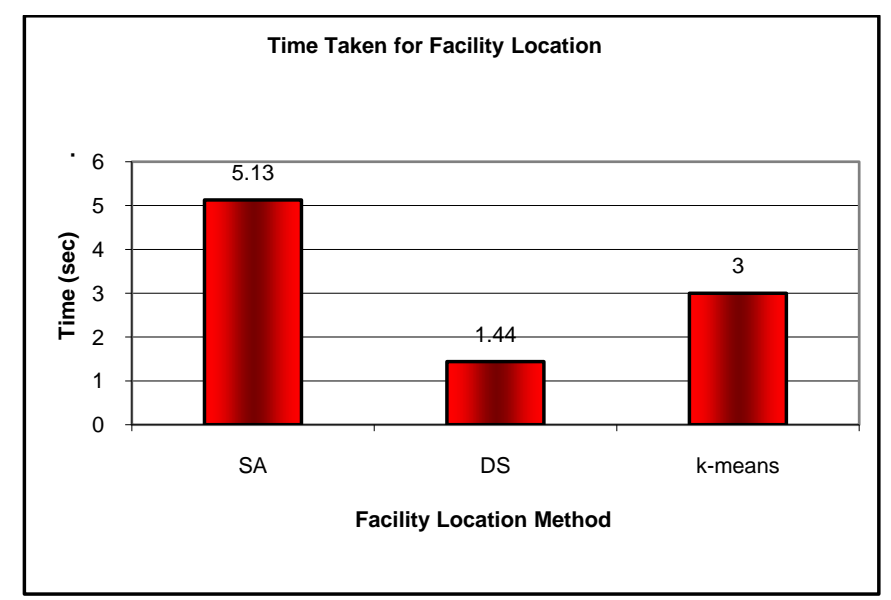

Figure 9. The Performance in Terms of CPU Time 


\section{CONCLUSION}

Facility location for logistics is a wide area for research. In this work it addressed the possibilities of using soft computing based models for facility location in logistics. It has used soft computing based clustering approach for Facility Location Problem \& Logistic Analysis.

As per the results, soft computing based optimization models successfully found optimum locations of facilities in considerably meaningful time limit.

In this work, it used a simple Euclidean distance function as a fitness function in the design of soft computing based location optimization model. But, there are much more constraints and parameters in a practical logistics problem that can be included in the design of the fitness function such as (1) travel time with respect to road type, (2) loading unloading time at DCs, (3) different more of travel times such as air travel time. This kind of more constraints and parameters can be included in future design of soft computing based optimization models. The future works will address these issues.

It has designed the proposed models as a single objective problem. But there are facility location and logistics situations where there may be more than one objective during optimization. Future works may address the design of soft computing based optimization models for multi objective optimization scenarios.

\section{REFERENCES}

[1] Erlenkotter, D., "A Dual-Based Procedure for the Uncapacitated Facility Location." Operations Research, Vol 26(6), pg. 992-1009, 1978.

[2] Francis, R.L., McGinnis, F.L., Jr., White, J.A., "Facility Layout and Location: An Analytical Approach." Prentice Hall, 2nd Ed., 1974.

[3] Juliana Karakaneva, "A LOCATION PROBLEM MODELING AND SOLVING", Trakia Journal of Sciences, Vol 1, No 4, pp 1-7, 2003,ISSN 1312-1723, Copyright (C) 2003 Trakia University

[4] HK Smith, G Laporte and PR Harper, "Locational analysis: highlights of growth to maturity", http://eprints.soton.ac.uk/68930/1/Locational_Analysis__Smith,_Laporte_and_Harper.doc

[5] Michael J. Bucci, Michael G. Kay, Donald P. Warsing†, Jeffrey A. Joines, "Metaheuristics for Facility Location Problems with Economies of Scale", IIE Transactions

[6] Michael J. Bucci, Ryan Woolard, Jeffrey Joines, Kristin Thoney, Russell E. King, "An Application of Heuristics Incorporating Economies of Scale to Facility Location Problems in Carpet Recycling"

[7] .Biehl, M., Prater, E., Realff, M.J., 2007, Assessing performance and uncertainty in developing carpet reverse logistics systems, Computers and Operations Research, $34,443-463$.

[8] .Brimberg, J.,Hansen, P., Mladenovic, N.,Taillard, E.D. ,2000, Improvements and Comparison of Heuristics for Solving the Uncapacitated Multisource Weber Problem, Operations Research, 48, 444-460.

[9] .Bucci, M.J., Kay, M.G., Warsing, D.P., Joines, J.A., 2009, Metaheuristics for Facility Location with Economies of Scale, Fitts Department of Industrial and Systems Engineering working paper, North Carolina
State University, Raleigh NC.

[10] Cooper, L., 1963, Location-allocation problems, Operations Research, 11, 331-343.

[11] CARE (Carpet America Recovery Effort) annual report, 2007, http://www.carpetrecovery.org/pdf/annual_report/07_CA RE-annual-rpt.pdf

[12] CARE (Carpet America Recovery Effort) network website, 2009 , http://www.carpetrecovery.org/pdf/reclamation_centers/ Carpet_Reclamation_Center s.pdf

[13] Daskin, M.S., 1995, Network and discrete location: models, algorithms, and applications, John Wiley and Sons, New York.

[14] De Brito, M.P., Dekker, R., Flapper, S.D.P., 2003, Reverse Logistics - a review of case studies, ERIM Report Series.

[15] Fleishchmann, M., Krikke, H.R., Dekker, R., Flapper, S.D.P., 2000, A characterization of logistics networks for product recovery, Omega, 28, 653-666.

[16] Louwers, D., Kip, B.J., Peters, E., Souren, F., Flapper, S.D.P., 1999, A facility location allocation model for reusing carpet, Computers and Industrial Engineering,

[17] Mirchandani, P.B., Francis, R.L., 1990, Discrete Location Theory, Wiley, NewYork.

[18] Realff, M.J., Ammons, J.C., Newton, D., 1999, Carpet Recycling: Determining the 38:3, 547-567.

[19] Realff, M., Systems Planning for Carpet Recycling. 2006, Recycling in Textiles, Editor Youjiang Wang, CRC Press.

[20] Shaju Varghese, Gladston Raj S, "A Genetic Algorithm Based Optimization Model for Facility Location in Logistic Network Design", International Journal of Applied Engineering Research" Vol 10, No. 69, pp-338344, 2005, ISSN 0973-4562.

\section{AUTHORS PROFILE}

Mr. Shaju Varghese received his M.Sc. (Maths), M.C.A., and M.Phil. in computer Science. Now working as Head of the Department of Computer Science at Baselios Poulose II Catholicos (B. P. C ) College, Piravom, Kerala, India. He was the Principal Investigator of the Minor Research Project "Computerized Facility Location Analysis In Rural Area Using Clustering", 2010, funded by Universities Grant Commission, India. His research interest includes Data Mining, Facility Location Problem, and Cyber Criminology. Currently he is pursuing Ph.D. in Computer Science at Bharathiar University, Tamilnadu, India.

Dr. Gladston Raj S. received his M.Sc (CS), M.Tech (Image Computing) and $\mathrm{PhD}$ in Computer Science from University of Kerala and Completed UGC-NET from University of Kerala and PGDCH (Computer hardware) from MicroCode, $\mathrm{He}$ is Now working as Head of the Department of Computer Science at Govt. College Nedumangad, Kerala, India. His area of interest includes Image Processing, Signal Processing, Datamining. He is providing research guidance for Ph.D scholars from different areas of research and has presented several invited talks in this areas of research. 